\section{Les cellules dendritiques inflammatoires}

Élodie Segura, Sebastian Amigorena

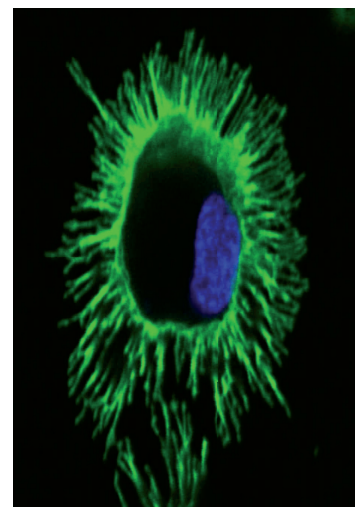

Inserm U932,

section recherche,

Institut Curie,

26, rue d'Ulm, 75005 Paris,

France.

elodie.segura@curie.fr

la prolifération et la dfférenciation dépendent de la cytokine Flt3L ou ligand de Flt3 (Fms-related tyrosine kinase 3 ligand) [1, 2].

Dans une situation d'inflammation, les populations de cellules dendritiques sont modifiées. Ainsi, lorsque l'inflammation est déclenchée par une infection ou un dommage tissulaire, on observe un recrutement massif de leucocytes (monocytes et neutrophiles) et la sécrétion de cytokines et de chimiokines inflammatoires [3]. De même, la migration des cellules dendritiques tissulaires vers les ganglions lymphatiques est augmentée, tandis qu'une nouvelle population de cellules dendritiques apparaît au site d'inflammation: les cellules dendritiques inflammatoires.

\section{Définition des cellules dendritiques inflammatoires}

Les cellules dendritiques inflammatoires ont tout d'abord été identifiées chez la souris dans un modèle d'infection cutanée par la bactérie Leishmania major [4]. Cette population de cellules qui expriment des molécules typiques des cellules dendritiques (molécules du CMH II et intégrine $(D 11 \mathrm{c}$ ) était absente avant l'infection, et apparaissait au site d'inflammation et dans les ganglions drainants après infection. Après cette description initiale, de nombreuses études ont montré chez la souris l'existence d'une population cellulaire comparable dans de nombreux cas d'inflammation causée par des pathogènes, qu'il s'agisse de bactéries (Listeria monocytogenes, Streptococcus pneumoniae, Salmonella typhimurium, etc.) [4-6], virus (virus de l'herpès, virus de la grippe, etc.) [6-8], levures ou parasites $[9,10]$. C'est également le cas dans des modèles d'inflammation expérimentale en l'absence de pathogène [11-15] ou encore dans des modèles de maladies inflammatoires (asthme, colite, arthrite rhumatoïde, sclérose en plaques) [6, 16-18]. Outre l'expression de CDIlc et du CMH II, les cellules dendritiques inflammatoires expriment les molécules CDIlb, Ly6C (lymphocyte 


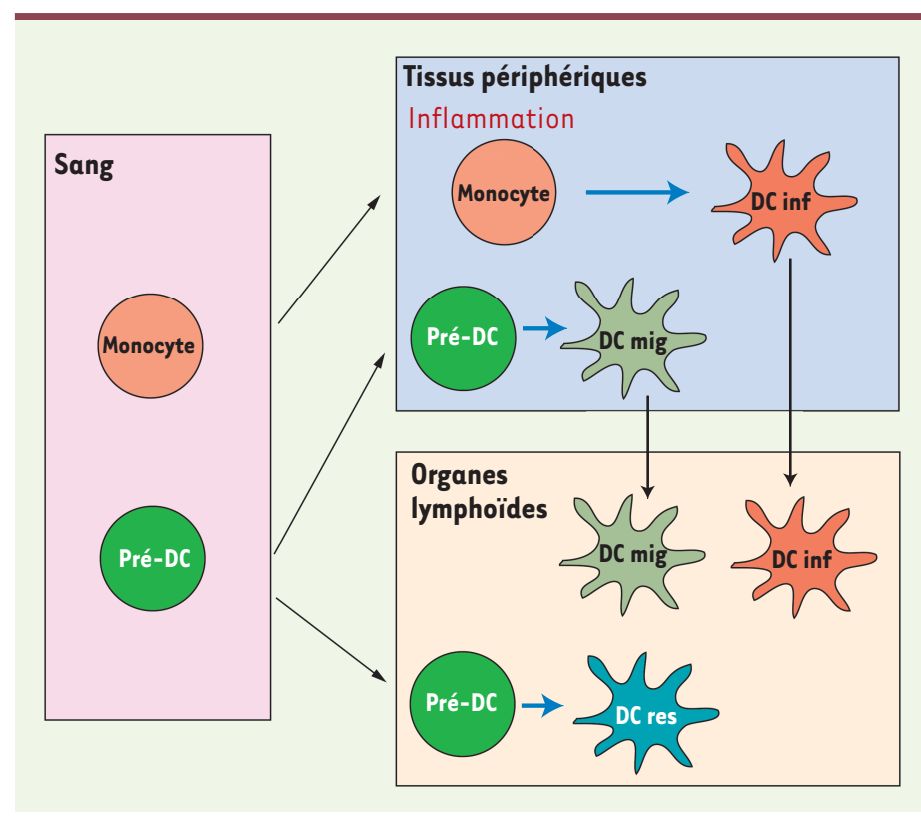

Figure 1. Ontogénie des cellules dendritiques. Les cellules dendritiques résidentes ( $D C$ res) et migratoires ( $D C$ mig) se différencient à partir de précurseurs hématopoïétiques communs, les pré-DC, qui circulent dans le sang. Au cours d'une inflammation, les monocytes sont recrutés sur le site de l'inflammation (tissus périphériques ou organes lymphoïdes) où ils se différencient en cellules dendritiques inflammatoires (DC inf). Le schéma illustre le cas d'une inflammation dans des tissus périphériques; les cellules dendritiques inflammatoires peuvent ensuite migrer vers les ganglions drainants. Flèche bleue : différenciation; flèche noire : migration.

antigen 6 complex), CD206/récepteur du mannose et CD64, molécules également exprimées à la surface des macrophages (ce qui peut entraîner une confusion dans certaines analyses) (Tableau I). Une étude récente a montré que l'expression du récepteur FcERI (IgG Fc receptor) était un très bon marqueur distinguant les cellules dendritiques inflammatoires des cellules dendritiques classiques et des macrophages [19].

Les cellules dendritiques inflammatoires se distinguent également des macrophages par leurs propriétés fonctionnelles. Outre leur capacité de stimuler efficacement les lymphocytes $T$, contrairement aux macrophages, les cellules dendritiques inflammatoires peuvent migrer vers les ganglions drainants, alors que les macrophages demeurent dans les tissus $[4,7,15,19]$. Comme pour les cellules dendritiques tissulaires, cette migration vers les ganglions drainants requiert le récepteur des chimiokines CCR7, expliquant l'absence des cellules dendritiques inflammatoires des ganglions chez les souris n'exprimant pas CCR7 $[15,19]$.

\section{Les cellules dendritiques inflammatoires dérivent de monocytes}

L'une des caractéristiques qui distingue les cellules dendritiques inflammatoires des cellules dendritiques classiques est leur origine : les premières se différencient in situ à partir de monocytes recrutés sur le site de l'inflammation, et non pas à partir des pré-DC comme les secondes (Figure 1). Différents groupes ont ainsi montré que, lors d'une inflammation, des monocytes injectés à des souris se différenciaient en cellules dendritiques inflammatoires $[4,6,9,12,19]$. L'utilisation de souris déficientes pour le récepteur de chimiokine CCR2, récepteur indispensable à la migration des monocytes, a permis d'aboutir à la même conclusion: l'absence de recrutement des monocytes sur le lieu d'inflammation compromet l'apparition des cellules dendritiques inflammatoires dont le nombre est alors sévèrement diminué $[5,8,9,13,20]$.

Si l'on sait que la formation des cellules dendritiques inflammatoires n'est pas affectée par l'absence du ligand de Flt3 [19], on connaît mal en revanche les molécules impliquées dans la différenciation des monocytes en cellules dendritiques inflammatoires. Une étude récente a montré que le récepteur du M-CSF (macrophage colony-stimulating factor) était requis pour la différenciation des cellules dendritiques inflammatoires, sans être impliqué dans le recrutement des monocytes sur le site d'inflammation [6].

\section{Le rôle des cellules dendritiques inflammatoires dans l'activation des lymphocytes T}

Les populations de cellules dendritiques résidentes et migratoires comportent chacune plusieurs sous-types aux propriétés différentes, en particulier en ce qui concerne la présentation des antigènes et l'activation des lymphocytes T. Ces observations ont conduit à un modèle de division du travail entre les populations de cellules dendritiques, chaque sous-type étant spécialisé dans certaines tâches. On commence à peine à comprendre la place des cellules dendritiques inflammatoires dans ce schéma.

Dans différents modèles d'inflammation, les cellules dendritiques inflammatoires directement purifiées à partir des tissus ou organes lymphoïdes peuvent activer ex vivo des lymphocytes $\mathrm{T} C D 4^{+}$spécifiques de l'antigène étudié. Cela indique que les cellules dendritiques inflammatoires ont capturé l'antigène in vivo et sont capables de le présenter efficacement $[4,7,9$, 14-16, 21]. II semblerait que le type de réponse induite (réponse Thelper de type 1, type 2, etc.) soit influencé par l'environnement inflammatoire. Ainsi, dans des modèles d'infection par Leishmania major ou Aspergillus fumigatus, dans lesquels la réponse Thelper de type 1 est dominante, les cellules dendritiques inflammatoires induisent la différenciation de lymphocytes $\mathrm{T} \mathrm{CD}^{+}$helper de type $1[4,9]$. En revanche, dans un 


\begin{tabular}{|c|c|c|c|c|c|c|}
\hline & $\begin{array}{c}\text { DC res } \\
\text { CD }^{+}\end{array}$ & $\begin{array}{l}\text { DC res } \\
\text { CD11 b }\end{array}$ & $\begin{array}{l}\text { DC mig } \\
\text { CD103 }\end{array}$ & $\begin{array}{l}\text { DC mig } \\
\text { CD11 b }\end{array}$ & $D C$ inf & $\begin{array}{c}\text { Macro- } \\
\text { phage }\end{array}$ \\
\hline CDllb & - & + & - & + & + & + \\
\hline Ly6C & - & - & - & - & + & + \\
\hline CD206 & - & - & - & + & + & + \\
\hline FcERI & - & - & - & - & + & - \\
\hline CD64 & - & - & - & - & + & ++ \\
\hline CDllc & ++ & ++ & ++ & ++ & + & $+/-$ \\
\hline CMH II & + & + & ++ & ++ & + & $+/-$ \\
\hline
\end{tabular}

Tableau I. Phénotype des populations de cellules dendritiques et macrophages murins. DC res : cellules dendritiques résidentes; DC mig : cellules dendritiques migratoires; $D C$ inf : cellules dendritiques inflammatoires.

modèle d'asthme, caractérisé par une réponse Thelper de type 2, les cellules dendritiques inflammatoires induisent la différenciation de lymphocytes T CD4 ${ }^{+}$helper de type $2[16,19]$.

Les cellules dendritiques inflammatoires peuvent également présenter des antigènes exogènes sur leurs molécules du $\mathrm{CMH}$ I, un processus appelé cross-presentation dans lequel sont spécialisées les cellules dendritiques résidentes $C D 8^{+}$et migratoires $C D 103^{+}$. En effet, les cellules dendritiques inflammatoires sont impliquées dans l'activation in vivo de lymphocytes $T$ CD8 ${ }^{+}$spécifiques de l'antigène étudié $[11,22]$. De plus, les cellules dendritiques inflammatoires directement purifiées des tissus ou organes lymphoïdes peuvent également activer ex vivo des lymphocytes $T C D 8^{+}$spécifiques après capture de l'antigène in vivo $[7,8,14,15]$. Concernant les mécanismes moléculaires de la crosspresentation, il a été montré que les cellules dendritiques inflammatoires utilisent une voie intracellulaire différente de celle qu'utilisent les cellules dendritiques résidentes CD8 ${ }^{+}$[14].

Les cellules dendritiques inflammatoires peuvent migrer dans les ganglions lymphatiques et $y$ transporter des antigènes capturés sur le site d'inflammation, comme c'est le cas dans l'infection à Aspergillus fumigatus [9]. Plusieurs études ont également montré un rôle prédominant des cellules dendritiques inflammatoires dans la présentation d'antigènes en périphérie. Ainsi, les cellules dendritiques inflammatoires qui se différencient lors d'une réactivation du virus de l'herpès HSV-1 stimulent les lymphocytes $T \mathrm{CD} 4^{+}$et $\mathrm{CD} 8^{+}$mémoires qui sont présents dans les tissus [22]. Dans le cas d'une inflammation causée par une infection avec le virus HSV-2, la stimulation des lymphocytes T effecteurs dans les tissus est très affectée par l'absence de cellules dendritiques inflammatoires [21], suggérant que ces cellules interagissent avec les lymphocytes $T$ effecteurs directement in situ. Dans un modèle d'asthme, les cellules dendritiques inflammatoires stimulent également les lymphocytes $\mathrm{T} C D 4^{+}$effecteurs présents dans les poumons, mais une forte dose d'allergène augmente la migration des cellules dendritiques inflammatoires vers les ganglions drainants où celles-ci activent également les lymphocytes T CD4 ${ }^{+}$dans les ganglions [19].
Ces études permettent de compléter le modèle de division du travail des populations de cellules dendritiques, conférant aux cellules dendritiques inflammatoires un rôle de stimulation des lymphocytes $T$ dans les tissus (Figure 2). Ceci permet une meilleure réponse effectrice lors d'une inflammation en stimulant les lymphocytes effecteurs in situ, et une activation plus rapide de la réponse immunitaire secondaire lorsque les cellules dendritiques inflammatoires stimulent des lymphocytes T mémoires résidant dans les tissus.

\section{Les cellules dendritiques inflammatoires humaines}

Chez l'homme, on retrouve des populations de cellules dendritiques résidentes et migratoires, constituées aussi de différentes sous-populations. Ainsi, dans le sang, la rate, les amygdales et les ganglions lymphatiques, on observe des cellules dendritiques résidentes $\mathrm{BDCAl}^{+}$ (blood dendritic cell antigen 1 ) et Clec $9 \mathrm{~A}^{+}$(C-type lectin domain family 9 member $A$ ). Dans la peau, on trouve trois populations de cellules dendritiques migratoires: les cellules dendritiques dermiques $\mathrm{CD} 1 \mathrm{a}^{+}$et $\mathrm{CD} 14^{+}$, et les cellules de Langerhans épidermiques [33].

Des cellules dendritiques épidermiques inflammatoires ont été décrites en 1996 dans la peau de patients présentant une dermatite atopique [23]. Ces cellules exprimaient des molécules de surface différentes de celles qu'expriment les cellules dendritiques dermiques et des cellules de Langerhans issues de peau saine. Cependant, leur caractérisation était incomplète et il n'a pas été déterminé si ces cellules représentaient une sous-population de cellules dendritiques ou une forme activée de cellules dendritiques de la peau. Des cellules exprimant les molécules du CMH II (HLA-DR) et CDIlc, présentes dans la peau de patients atteints de psoriasis et absentes dans la peau saine, ont également été observées et nommées cellules dendritiques dermiques inflammatoires [24]. Néanmoins, ces cellules ont ensuite été identifiées comme étant similaires à des cellules du sang exprimant $\mathrm{CD}_{16}{ }^{+}$et la 6 -sulfo LacNAc (sous-population de monocytes) $[25,26]$. De plus, les caractéristiques de ces cellules les rapprochent plus des macrophages que des cellules dendritiques [27]. Ainsi, alors que la capacité de différenciation de monocytes humains en cellules dendritiques était connue depuis longtemps et largement utilisée pour l'obtention de cellules dendritiques in vitro [28], la dérivation in vivo de cellules dendritiques inflammatoires humaines à partir de monocytes n'avait pas été identifiée jusqu'à récemment.

Nous avons analysé les cellules dendritiques présentes dans deux liquides inflammatoires humains : les ascites 


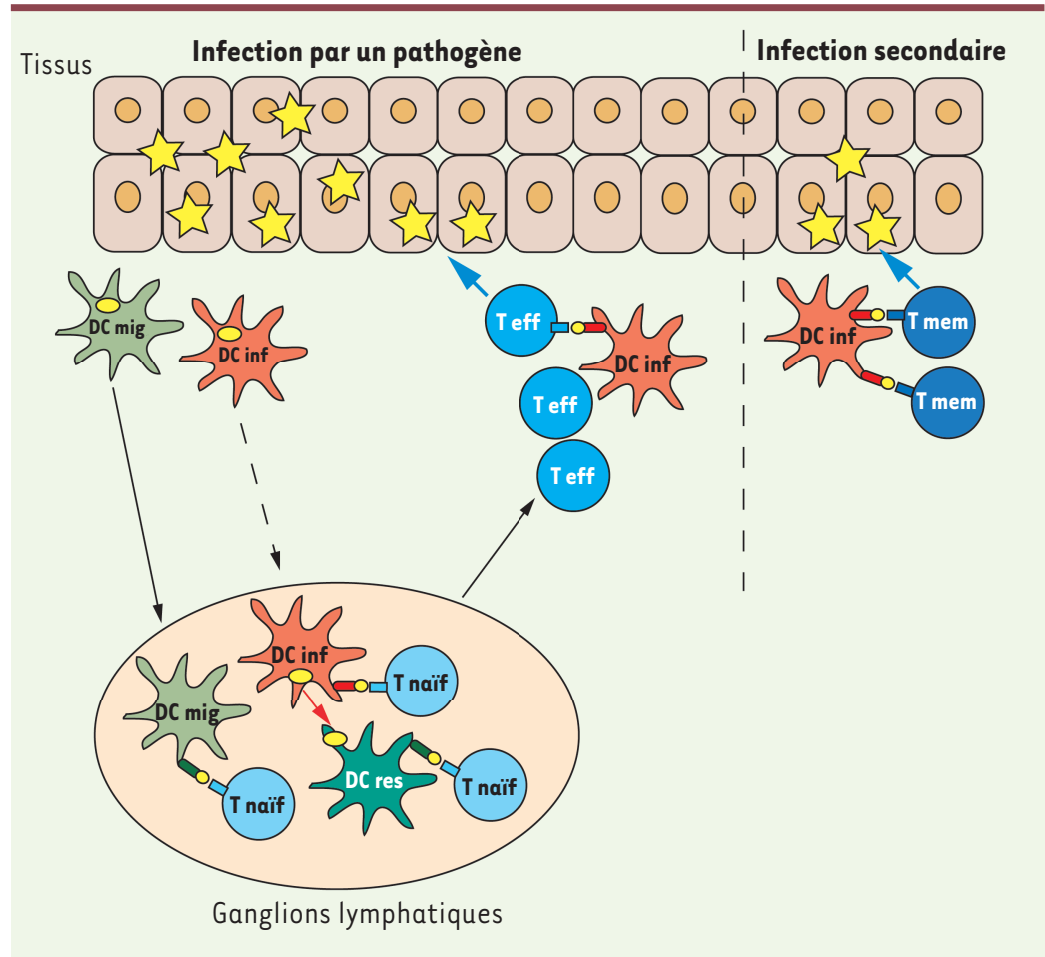

Figure 2. Rôle des cellules dendritiques inflammatoires dans les réponses immunitaires. Lors d'une inflammation causée par une infection par un pathogène, les cellules dendritiques migratoires du tissu capturent l'antigène et migrent vers les ganglions lymphatiques drainants où elles peuvent activer des lymphocytes $T$ naïfs ( $T$ naïfs) spécifiques du pathogène. En cas de forte inflammation, les cellules dendritiques inflammatoires peuvent également migrer vers les ganglions où elles transfèrent l'antigène capturé en périphérie à des cellules dendritiques résidentes; elles peuvent aussi le présenter directement aux lymphocytes $T$ naïfs. Les lymphocytes $T$ se différencient ensuite en cellules $T$ effectrices ( $T$ eff) qui peuvent être stimulées à nouveau dans les tissus par des cellules dendritiques inflammatoires. Lors d'une seconde infection par le même pathogène, les cellules dendritiques inflammatoires peuvent activer directement in situ des lymphocytes T mémoires (T mem) spécifiques du pathogène résidant dans les tissus.

associées à des tumeurs et le liquide synovial arthritique. Dans les deux cas, nous avons identifié une population de cellules dendritiques inflammatoires distincte des cellules dendritiques classiques, et distincte des macrophages inflammatoires présents dans les mêmes liquides [29]. De plus, nous avons montré par une analyse transcriptomique que les cellules dendritiques inflammatoires humaines expriment une signature génique proche de celle des cellules dendritiques dérivées in vitro de monocytes en présence de GM-CSF (granulocyte macrophage colony-stimulating factor) et d'IL-4 (interleukine-4). Cela suggère que, comme chez la souris, les cellules dendritiques inflammatoires humaines dérivent également de monocytes.

Les cellules dendritiques inflammatoires humaines sont caractérisées par l'expression de molécules exprimées par d'autres populations de cellules dendritiques - CMH II (HLA-DR), CDllc, BDCAl, CDla, FcERI -, mais également de molécules habituellement considérées comme des marqueurs de macrophages, telles que CD206, CD14 (récepteur du lipopolysaccharide) and CD11b [29-31]. En ce qui concerne leurs propriétés fonctionnelles, nous avons montré que les cellules dendritiques inflammatoires purifiées d'ascites tumorales ou de liquide synovial arthritique - deux situations où prédominent des réponses helper de type 17 [32] - induisent ex vivo la différenciation de lymphocytes $T$ $\mathrm{CD}^{+}$helper de type 17 [29]. Les réponses induites dans d'autres types d'inflammation restent à étudier.

\section{Conclusion}

Des études récentes ont montré l'existence, chez la souris, d'une population de cellules dendritiques spécifique des environnements inflammatoires, qui se distingue en particulier des autres populations de cellules dendritiques par leur origine et leur capacité à stimuler les lymphocytes $T$ directement dans les tissus. Ces cellules dendritiques inflammatoires ont également été décrites chez l'homme et pourraient représenter une cible thérapeutique potentielle dans les maladies inflammatoires. $\diamond$

\section{SUMMARY}

Inflammatory dendritic cells

Dendritic cells are a rare and heterogeneous population of professional antigen-presenting cells. Several murine dendritic cell subpopulations have been identified that differ in their phenotype and functional properties. In the steady state, committed dendritic cell precursors differentiate into lymphoid organ-resident dendritic cells and migratory tissue dendritic cells. During inflammation appears an additional dendritic cell subpopulation that has been termed «inflammatory dendritic cells ». Inflammatory dendritic cells differentiate in situ from monocytes recruited to the site of inflammation. Here, we discuss how mouse inflammatory dendritic cells differ from macrophages and from other dendritic cell populations. Finally, we review recent work on human inflammatory dendritic cells. $\diamond$

\section{LIENS D'INTÉRÊT}

Les auteurs déclarent n'avoir aucun lien d'intérêt concernant les données publiées dans cet article. 


\section{RéFÉRENCES}

1. Steinman RM, Idoyaga J. Features of the dendritic cell lineage. Immunol Rev $2010 ; 234: 5-17$.

2. Miller JC, Brown BD, Shay T, et al. Deciphering the transcriptional network of the dendritic cell lineage. Nat Immunol $2012 ; 13: 888-99$.

3. Medzhitov R. Origin and physiological roles of inflammation. Nature $2008 ; 454: 428-35$.

4. Leon B, Lopez-Bravo M, Ardavin C. Monocyte-derived dendritic cells formed at the infection site control the induction of protective Thelper 1 responses against Leishmania. Immunity 2007 ; 26 : 519-31.

5. De Trez C, Magez S, Akira S, et al. iNOS-producing inflammatory dendritic cells constitute the major infected cell type during the chronic Leishmania major infection phase of C57BL/6 resistant mice. PLoS Pathog $2009 ; 5$ : el000494.

6. Greter M, Helft J, Chow A, et al. GM-CSF Controls nonlymphoid tissue dendritic cell homeostasis but is dispensable for the differentiation of inflammatory dendritic cells. Immunity $2012 ; 36$ : 1031-46.

7. Ballesteros-Tato A, Leon B, Lund FE, Randall TD. Temporal changes in dendritic cell subsets, crosspriming and costimulation via CD70 control CD8(+) T cell responses to influenza. Nat Immunol $2010 ; 11: 216-24$.

8. Aldridge JR Jr, Moseley CE, Boltz DA, et al. TNF/iNOS-producing dendritic cells are the necessary evil of lethal influenza virus infection. Proc Natl Acad Sci USA 2009 ; 106 : 5306-11.

9. Hohl TM, Rivera A, Lipuma L, et al. Inflammatory monocytes facilitate adaptive CD4 T cell responses during respiratory fungal infection. Cell Host Microbe $2009 ; 6: 470-81$.

10. Guilliams M, Movahedi K, Bosschaerts T, et al. IL-10 dampens TNF/inducible nitric oxide synthaseproducing dendritic cell-mediated pathogenicity during parasitic infection. J Immunol 2009 ; $182: 1107-18$.

11. Le Borgne M, Etchart N, Goubier A, et al. Dendritic cells rapidly recruited into epithelial tissues via CCR6/CCL20 are responsible for CD8 ${ }^{+}$T cell crosspriming in vivo. Immunity $2006 ; 24: 191-201$.

12. Naik SH, Metcalf $D$, van Nieuwenhuijze A, et al. Intrasplenic steady-state dendritic cell precursors that are distinct from monocytes. Nat Immunol 2006; 7:663-71.

13. Nakano $\mathrm{H}$, Lin KL, Yanagita $M$, et al. Blood-derived inflammatory dendritic cells in lymph nodes stimulate acute Thelper type 1 immune responses. Nat Immunol $2009 ; 10: 394-402$

14. Segura $\varepsilon$, Albiston AL, Wicks IP, et al. Different cross-presentation pathways in steady-state and inflammatory dendritic cells. Proc Natl Acad Sci USA 2009; 106 : 20377-81.

15. Langlet C, Tamoutounour S, Henri S, et al. CD64 expression distinguishes monocyte-derived and conventional dendritic cells and reveals their distinct role during intramuscular immunization.J Immunol $2012 ; 188: 1751-60$.

16. Hammad H, Plantinga M, Deswarte $K$, et al. Inflammatory dendritic cells - not basophils - are necessary and sufficient for induction of Th2 immunity to inhaled house dust mite allergen. J Exp Med $2010 ; 207: 2097-111$.

17. Siddiqui KR, Laffont S, Powrie F. $\varepsilon$-cadherin marks a subset of inflammatory dendritic cells that promote T cell-mediated colitis. Immunity $2010 ; 32$ : 557-67.

18. Campbell IK, van Nieuwenhuijze A, Segura $\varepsilon$, et al. Differentiation of inflammatory dendritic cells is mediated by NF-\{kappa\}Bl-dependent GM-CSF production in CD4 T cells. J Immunol 2011 ; $186: 5468-77$.

19. Plantinga M, Guilliams M, Vanheerswynghels $M$, et al. Conventional and monocyte-derived CD1lb dendritic cells initiate and maintain Thelper 2 cell-mediated immunity to house dust mite allergen. Immunity $2013 ; 38: 322-35$.

20. Osterholzer JJ, Chen GH, Olszewski MA, et al. Accumulation of CDIlb+ lung dendritic cells in response to fungal infection results from the CCR2-mediated recruitment and differentiation of Ly-6Chigh monocytes. I Immunol 2009; 183 : 8044-53.
21. lijima N, Mattei LM, Iwasaki A. Recruited inflammatory monocytes stimulate antiviral Thl immunity in infected tissue. Proc Natl Acad Sci USA 2011 ; 108 : 284-9

22. Wakim LM, Waithman J, van Rooijen N, et al. Dendritic cell-induced memory T cell activation in nonlymphoid tissues. Science $2008 ; 319$ : 198-202.

23. Wollenberg A, Kraft S, Hanau D, Bieber T. Immunomorphological and ultrastructural characterization of Langerhans cells and a novel, inflammatory dendritic epidermal cell (IDEC) population in lesional skin of atopic eczema. J Invest Dermatol 1996 ; 106 : 446-53.

24. Zaba LC, Fuentes-Duculan J, Eungdamrong NJ, et al. Psoriasis is characterized by accumulation of immunostimulatory and Th1/Th17 cellpolarizing myeloid dendritic cells. J Invest Dermatol 2009 ; 129 : 79-88.

25. Hansel A, Gunther C, Ingwersen J, et al. Human slan (6-sulfo LacNAc) dendritic cells are inflammatory dermal dendritic cells in psoriasis and drive strong TH17/THI T-cell responses. J Allergy Clin Immunol 2011 ; 127 : 787-94 el-9.

26. Cros J, Cagnard N, Woollard K, et al. Human CD14dim monocytes patrol and sense nucleic acids and viruses via TLR7 and TLR8 receptors. Immunity $2010 ; 33: 375-86$

27. Zaba LC, Fuentes-Duculan J, Eungdamrong NJ, et al. Identification of TNFrelated apoptosis-inducing ligand and other molecules that distinguish inflammatory from resident dendritic cells in patients with psoriasis.J Allergy Clin Immunol $2010 ; 125: 1261-8$ e9.

28. Sallusto F, Lanzavecchia A. Efficient presentation of soluble antigen by cultured human dendritic cells is maintained by granulocyte/macrophage colony-stimulating factor plus interleukin 4 and downregulated by tumor necrosis factor alpha. J Exp Med 1994 ; 179 : 1109-18.

29. Segura $\varepsilon$, Touzot $M$, Bohineust $A$, et al. Human inflammatory dendritic cells induce Th17 cell differentiation. Immunity $2013 ; 38: 336-48$.

30. Wollenberg A, Mommaas M, Oppel T, et al. Expression and function of the mannose receptor CD206 on epidermal dendritic cells in inflammatory skin diseases. J Invest Dermatol 2002 ; 118 : 327-34

31. Guttman-Yassky E, Lowes MA, Fuentes-Duculan J, et al. Major differences in inflammatory dendritic cells and their products distinguish atopic dermatitis from psoriasis. J Allergy Clin Immunol 2007 ; 119 : 1210-7.

32. Leung-Theung-Long S, Guerder S. Les cellules Th17 - Une nouvelle population de cellules T CD4 effectrices pro-inflammatoires. Med Sci(Paris) $2008 ; 24: 972-6$.

33. Le Borgne M, Dubois B, Kaiserlian D. Cellules dendritiques des muqueuses de la peau. Med Sci (Paris) 2007 ; $23: 819-25$.

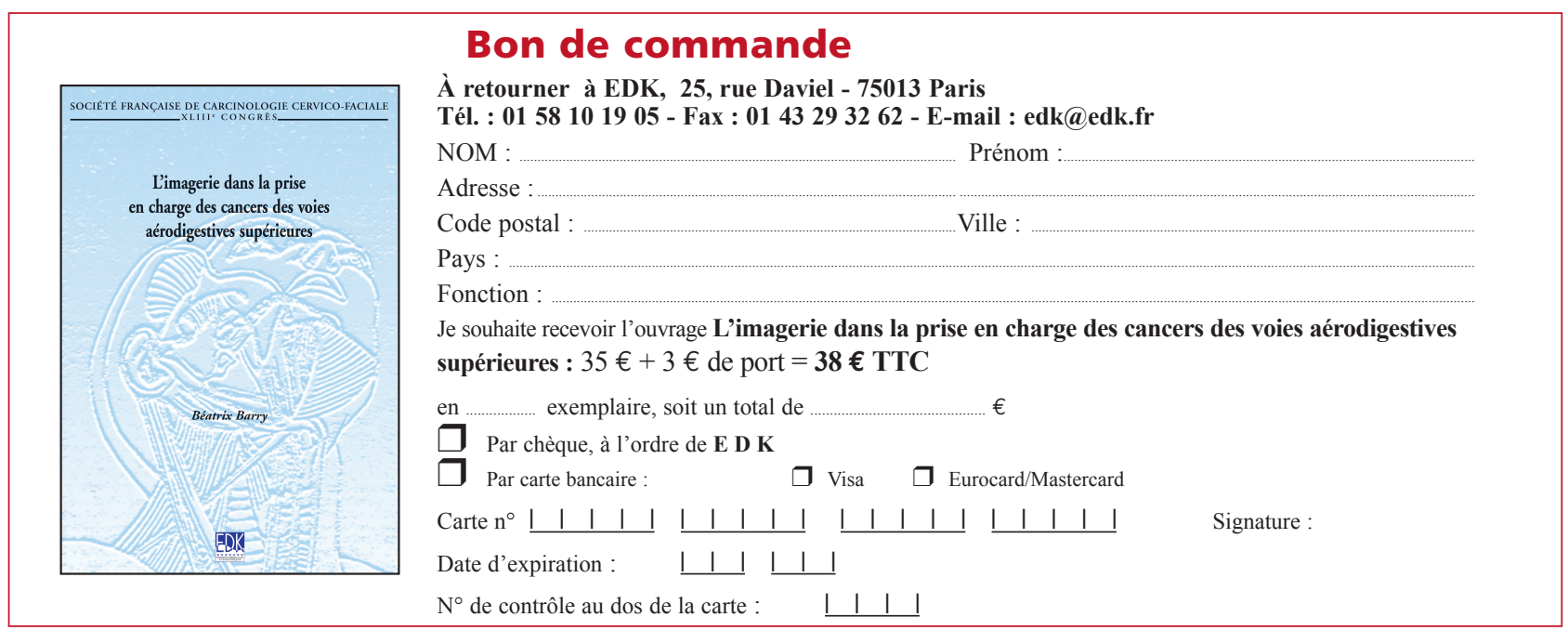

